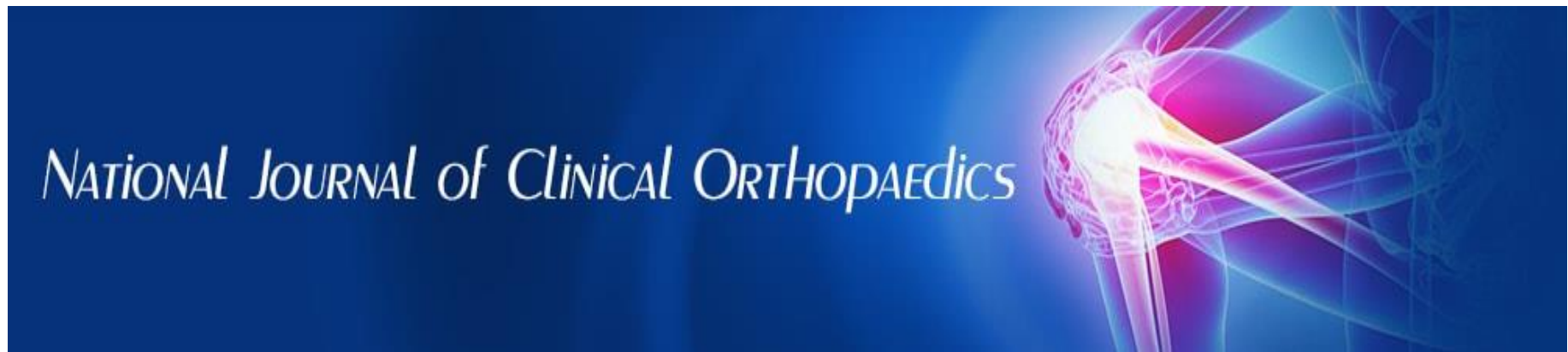

ISSN (P): 2521-3466

ISSN (E): 2521-3474

(C) Clinical Orthopaedics www.orthoresearchjournal.com 2021; 5(3): 28-32

Received: 18-05-2021

Accepted: 24-06-2021

Dr. Sanjan Das

Assistant Professor, Department of Orthopaedic Surgery, Gauhati Medical College and Hospital,

Guwahati, Assam, India

Dr. Siddhartha Rai

Senior Resident, Department of Orthopaedic Surgery, Gauhati

Medical College and Hospital,

Guwahati, Assam, India

Dr. Herman Conrad Frank Professor, Department of Orthopaedic Surgery, Gauhati Medical College and Hospital, Guwahati, Assam, India

Dr. Dhruba Narayan Borah Assistant Professor, Department of Orthopaedic Surgery, Gauhati Medical College and Hospital, Guwahati, Assam, India
Corresponding Author: Dr. Siddhartha Rai Senior Resident, Department of Orthopaedic Surgery, Gauhati Medical College and Hospital, Guwahati, Assam, India

\section{The Latarjet procedure for the treatment of recurrent anterior shoulder instability: A case series}

\author{
Dr. Sanjan Das, Dr. Siddhartha Rai, Dr. Herman Conrad Frank and Dr. \\ Dhruba Narayan Borah
}

DOI: https://doi.org/10.33545/orthor.2021.v5.i3a.288

\begin{abstract}
Background: Recurrence of glenohumeral instability is common in the presence of osseous defect of glenoid after an arthroscopic Bankart's repair or open capsulolabral repair. The Latarjet's procedure that involves transfer of the coracoid to tuck the glenoid bone defect is a popular surgical procedure and this study was undertaken to evaluate the efficacy of Latarjet's procedure in the management of recurrent shoulder dislocations after a failed index surgical procedure other than Latarjet's procedure.

Materials and Methods: A total of 5 patients were included in the study ( 3 male; 2 female). All the patients were operated using the Latarjet's procedure and a follow up was done at regular intervals. The evaluation at follow up included serial radiographs along with outcome assessment parameters such as subjective shoulder score, Constant-Murley score and VAS score.

Results: There were no cases of redislocations or subluxations at the end of follow up. No revision surgery was needed. The mean shoulder value improved from $61 \%$ to $83 \%$ postoperatively at 1 year follow up $((p<0.001))$ and the relative Constant -Murley score improved from $75 \%$ preoperatively to $85 \%$ postoperatively at the end of 1 year of follow up $>0.05)$.

Conclusion: Coracoid transfer as described by Latarjet can effectively restore anterior glenohumeral shoulder stability if previous operation(s) have failed to do so.
\end{abstract}

Keywords: Glenohumeral instability, anterior shoulder instability, recurrent shoulder instability, coracoid transfer, Latarjet's procedure, Bankart's repair, constant-murley score

\section{Introduction}

Anterior glenohumeral instability is reported to be as high as 3\% per year in the young athletic population ${ }^{[1]}$. Conservative approach to management along with arthroscopic treatment of glenohumeral instability have been associated with high rates of recurrence, particularly in the setting of glenohumeral osseous defects ${ }^{[1]}$. Latarjet procedure has become one of the fundamentals in shoulder-stabilising surgery, introduced by Latarjet in 1954 in the journal lyon chirurgical ${ }^{[2]}$. Through a deltopectoral approach, coracoid process and soft tissue are attached to the anteriorinferior border of the glenoid. The Latarjet procedure has been well described for restoring glenohumeral stability in patients with $>25 \%$ glenoid bone loss ${ }^{[3]}$. Coracoid process transfer for the treatment of recurrent glenohumeral dislocations is a safe and reliable procedure ${ }^{[4]}$; this stabilizes the shoulder through a triple mechanism which uses the conjoint tendon as a sling and the coracoid process as a bony block, while repairing the capsule via fixation to the coracoacromial ligament ${ }^{[5]}$. The Latarjet is a well-established treatment option with good evidence for favourable long term outcomes ${ }^{[6]}$. Intraoperative complications such as graft malpositioning, neurovascular injury, and graft fracture can all be mitigated with meticulous surgical technique and understanding of the local anatomy ${ }^{[7]}$. With proper patient selection and systematic surgical technique, instability can be eliminated without loss of external rotation in more than $98 \%$ of cases ${ }^{[8]}$. Our study was undertaken with the objective of determining the efficacy of Latarjet procedure in the management of recurrent shoulder dislocations after a failed index surgical procedure other than Latarjet's procedure. 


\section{Materials and Methods}

\subsection{Patients}

The study was conducted in the Department of Orthopaedics, Gauhati medical college \& hospital, Guwahati during 2019(april-october) after taking institutional ethical clearance. A total of 5 patients ( 3 male, 2 female) were included in the study after obtaining informed and written consents. Patients were followed up for a period of 1 year (table 1).

\subsection{Indication of Latarjet procedure}

a. Clinical finding of recurrence of anterior shoulder instability after a previously failed surgical repair.

b. CT scan demonstrating more than $20 \%$ bone loss of glenoid

c. Less than grade II fatty infiltration of subscapularis (Goutallier et al.) ${ }^{[9]}$

\subsection{Preoperative evaluation}

An arthro -CT scan was performed to assess the glenoid bone defect (figure 1). Hill-Sachs lesion and presence of osteoarthritis was assessed in accordance to classification system proposed by Samilson and Prieto ${ }^{[10]}$

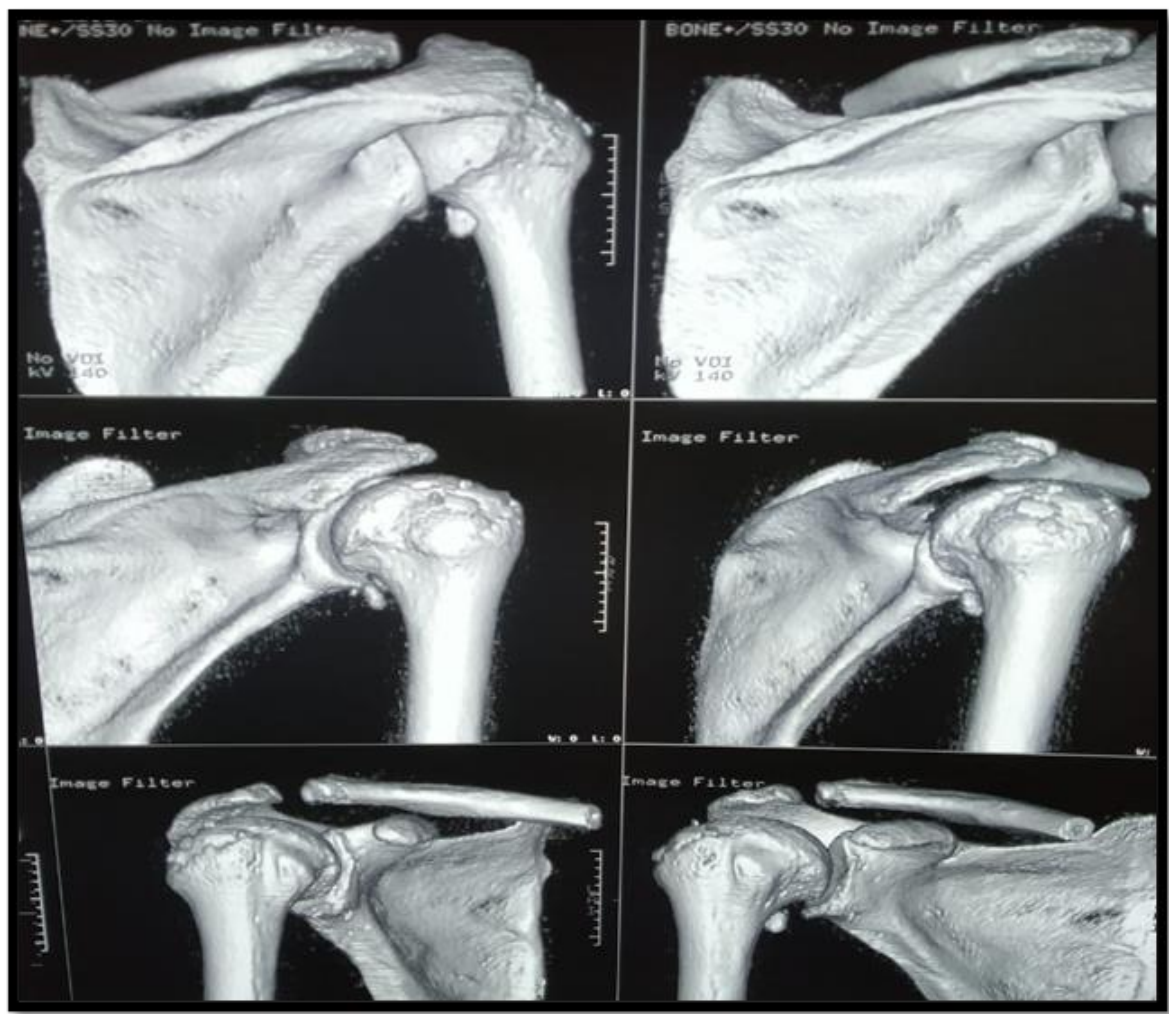

Fig 1: CT scan demonstrating Glenoid bone loss

Table 1: Patients included in the study

\begin{tabular}{|c|c|c|c|c|c|}
\hline Cases & $\begin{array}{c}\text { Age at occurrence of } \\
\text { recurrent instability(years) }\end{array}$ & Sex & $\begin{array}{c}\text { Cause of recurrent } \\
\text { instability }\end{array}$ & Extremity & Primary procedure \\
\hline Case 1 & 23 & Male & Major trauma & Right & Arthroscopic Bankart repair \\
\hline Case 2 & 20 & Female & Major trauma & Right & Open capsulolabral repair \\
\hline Case 3 & 37 & Female & Minor Trauma & Right & Arthroscopic Bankart repair \\
\hline Case 4 & 35 & Male & Major trauma & Left & Arthroscopic Bankart repair \\
\hline Case 5 & 41 & Male & No trauma & Right & Open capsulolabral repair \\
\hline
\end{tabular}

\subsection{Surgical technique ${ }^{[11]}$ (figure 2)}

All the patients were operated in beach chair position after induction of general anesthesia with padding of all the bony prominences. The standard deltopectoral approach was used. The coracoid process was palpated and a $5-7 \mathrm{~cm}$ incison was made from the tip of coracoid to deltopectoral interval distally. Blunt dissection of soft tissue was carried out and clavipectoral fascia was opened in line with skin incision. Cephalic vein was protected by retracting it laterally. After obtaining sufficient exposure of coracoid process, abduction and external rotation of shoulder was carried out to improve visulaisation of coracoacromial ligament. The coracoacromial ligament was transected $1 \mathrm{~cm}$ from its insertion. The pectoralis minor tendon was thereafter stripped of its insertion from medial aspect of coracoid aided by adduction and internal rotation of shoulder.
The musculocutaneous and axillary nerve was identified and protected throughout the exposure of coracoid. An oscillating saw was used to perform a medio-lateral osteotomy of the coracoid slightly anterior to insertion of coraco-clavicular ligament at coracoid base. After the completion of osteotomy the coracoid graft was held with the help of grasping forceps and the coracohumeral ligament was released to cap off the coracoid harvest. The coracoid graft was prepared then for transfer by performing debridement of attached soft tissues. Meticulous care was taken during graft preparation to preserve the CAL stump as well as the conjoint tendon insertion and its adjacent blood supply to the coracoid graft. After completion of coracoid graft harvest, exposure of glenoid was carried out. The junction of upper two third and lower one third of subscapularis was identified and splitted. A L-shaped capsulotomy is performed- 
superior first at the superior glenoid and then approximately $1 \mathrm{~cm}$ medial to the glenoid rim. The antero-inferior glenoid rim was abraded with high speed burr to prepare the bed for coarcoid graft. The coracoid graft was thereafter transferred with the longitudinal axis of coracoid graft positioned superoinferiorly along the glenoid neck flush with the articular surface. Two kirschner wires were used for temporary fixation of graft and definitive fixation was done using $3.5 \mathrm{~mm}$ bicortical screws in the antero-posterior direction. Capsular and subscapularis muscle repair was carried out. Wound was copiously irrigated and closed in a layered fashion. A sterile post operative dressing was done followed by application of well padded abduction sling.
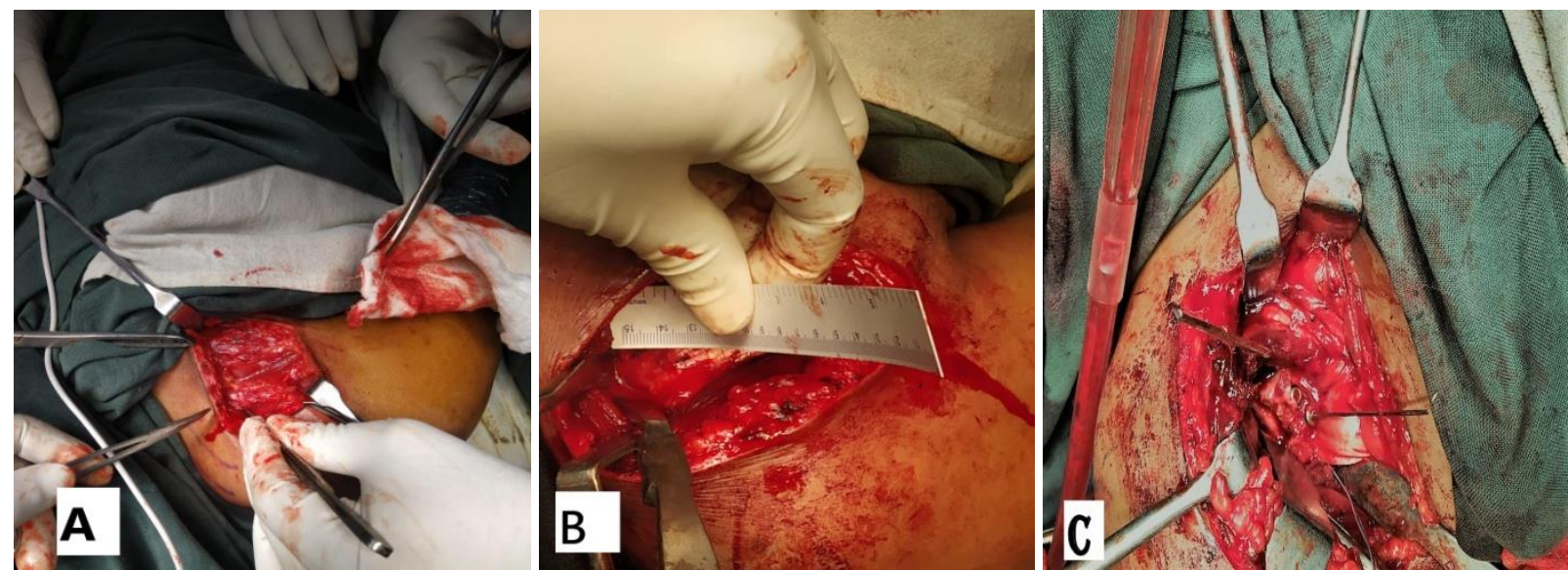

Fig 2(A-C)A: Exposure of shoulder through the standard deltopectoral approach B: Coracoid graft harvest C: Fixation of coracoid graft to glenoid bone defect using $3.5 \mathrm{~mm}$ cortical screws

\subsection{Follow up}

All the patients were followed up for a minimum of 1 year. Follow up was done at 2 weeks, 6 weeks, 3 months, 6 months and 1 year. Sutures were removed after 2 weeks and a sling was used for 6 weeks post-operatively. Patients were allowed to perform gentle passive, active, and active-assisted shoulder range of motion in the scapular plane. Radiographs were performed at follow up and active strengthening exercises were allowed once osseous healing was visualized (figure 3 ).

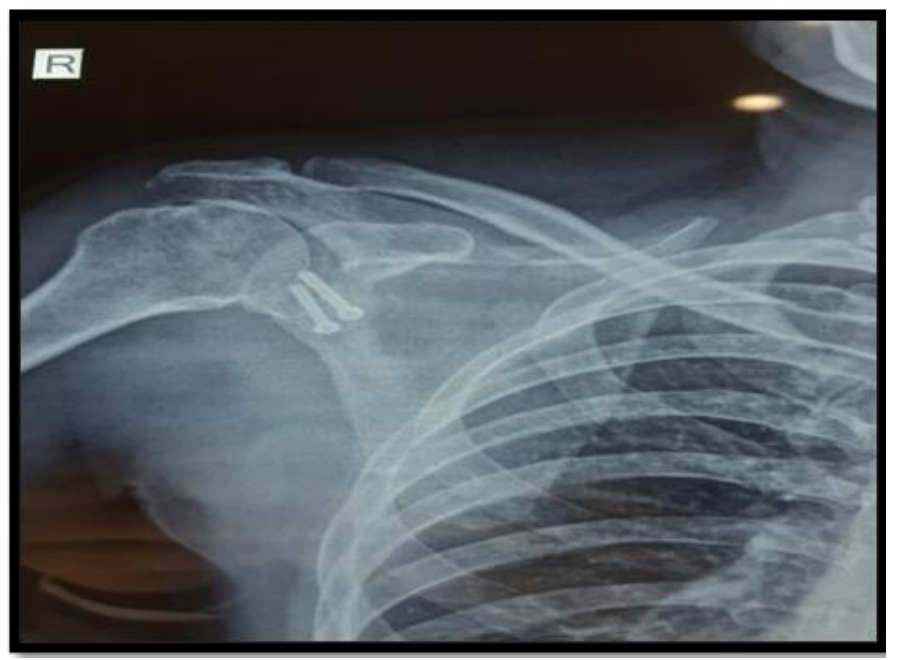

Fig 3: Follow up X-ray at 6 months

\subsection{Outcome assessment}

Clinical results were assessed with use of the subjective shoulder value $^{[12]}$. In this score, a normal shoulder is assigned a value of $100 \%$ and a patient subjectively assigns a comparatively value to the affected shoulder as a percentage of normal. Assesment also included the Constant -Murley score ${ }^{[13]}$ alongwith VAS for pain measurement. Goniometer was used to assess the active shoulder motion.

\section{Results}

A total number of 5 patients were included in our study. 3 were male $(60 \%)$ and 2 were female $(40 \%)$. The mean age at recurrent dislocation was 31.2 years. The right extremity was involved in 4 out of 5 patients $(80 \%)$. Arthroscopic Bankart's repair was the index surgical procedure in $60 \%$ of the patients whereas in $40 \%$ of the patients the primary surgical procedure was an open capsulolabral repair.

None of the five shoulder operated sustained a redislocation of gleno humeral joint. The mean shoulder value improved from $61 \%$ to $83 \%$ postoperatively. The relative Constant- Murley score was $75 \%$ preoperatively and $85 \%$ postoperatively. The VAS score was 6.2 preoperatively and improved to 2.9 postoperatively. Active external rotation decreased by 5 degrees. The preoperative and postoperative outcome measurements are summarized in table 2. The activity levels and range of movements are summarized in table 3. All the patients returned to their work status and difference between number of patients who had glenohumeral arthritis before the procedure and after the operation was not significant. We encountered one case of delayed wound healing and one case of frozen shoulder that resolved after antibiotic therapy and physical therapy respectively.

Table 2: Summary of Outcome assessment in the Preoperative and Postoperative period

\begin{tabular}{|c|c|c|c|}
\hline Criteria & Pre-operative & Post-operative follow up (1 year) & p-value \\
\hline Subjective shoulder value (\%) & 61.4 & 83.2 & $<0.001$ \\
\hline Constant-Murley score (\%) & 75.4 & 85.1 & $>0.05$ \\
\hline VAS & 6.2 & 2.9 & $<0.001$ \\
\hline
\end{tabular}


Table 3: Activity level and range of movements before and after the Latarjet's procedure

\begin{tabular}{|c|c|c|c|}
\hline Criteria & Pre-operative & Post-operative follow up (1 year) & p-value \\
\hline Activity level (points) & 5.9 & 8.3 & $<0.001$ \\
\hline Flexion (points) & 9.2 & 9.5 & $>0.05$ \\
\hline Abduction (points) & 9.1 & 9.6 & $>0.05$ \\
\hline External rotation (deg) & 56.4 & 51.2 & $<0.05$ \\
\hline Power (points) & 14.1 & 13.9 & 0.797 \\
\hline
\end{tabular}

\section{Discussion}

The goal of our study was to evaluate the efficacy of the Latarjet procedure in the treatment of previously failed repair of anterior shoulder instability. Our study demonstrates that stability is restored in most cases irrespective of the presence of hill sachs lesion.

The results of our study is comparable to the studies conducted in yesteryears. Allain et al. reported no dislocation in a follow up of 14 years after Latarjet repair in 58 patients ${ }^{[14]}$. Hovelius et al. reported a dislocation of $3.4 \%$ in a long term follow up in study involving 118 shoulders ${ }^{[15]}$. A large retrospective multicentre study in France in 2000 concluded that results of primary Bankart repair and Latarjet procedure is comparable with a subjectively better outcome after Latarjet procedure ${ }^{[16]}$. A retrospective study conducted by Schmid et al. involving 49 patients showed no cases of redislocation after Latarjet and comaparble results with our study ${ }^{[17]}$. Currently, the presence of osseous glenoid defect is the primary indication for the use of a bone augmentation procedure such as the Latarjet's coracoid transfer.

Positioning of the coracoids graft along the glenoid rim is paramount to success of Latarjet's procedure ${ }^{14-16}$. Previous studies have shown optimal placement of coracoid in $41 \%$ to $71 \%$ of the cases. Schmid et al. reported accurate graft placement in $73 \%$ cases ${ }^{[17]}$. Studies have concluded that suboptimal graft placement is associated with accentuation of pre existing arthritis alongwith new development of arthritis [16, 17]

Clinically relevant loss of shoulder movement was not seen in our case series in concurrence with previous studies ${ }^{[18,19]}$. There was a significant improvement in subjective shoulder score; however the Constant- Murley score improved only slightly owing to the fact that it is relatively insensitive to glenohumeral instability.

There were a number of limitations in our study. The sample size was small and the follow up period was not long enough. We did not compare the Latarjet procedure with other procedures used to restore stability after glenohumeral instability recurrence following previous surgical treatment. We did not address the treatment of recurrence of instability associated with insufficiency of the subscapularis tendon and muscle.

\section{Conclusion}

Based on the findings of our study we conclude that the Latarjet's procedure is a satisfactory revision procedure to restore the glenohumeral stability after failed primary operative repair irrespective of presence of Hill-sach's lesion with an intact subscapularis muscle.

\section{Declaration of conflicting interests}

The author(s) declared no potential conflicts of interest with respect to the research, authorship, and/or publication of this article.

\section{Funding}

The author(s) received no financial support for the research, authorship, and/or publication of this article

\section{References}

1. Mchale KJ, Sanchez G, Lavery KP, Rossy WH, Sanchez A, Ferrari MB. provencher mt. Latarjet technique for treatment of anterior shoulder instability with glenoid bone loss. Arthroscopy techniques 2017;6(3):e791-9.

2. Van Der Linde JA, Wessel RN, Trantalis JN, Van Den Bekerom MP. Review of latarjet (1954) on the treatment of recurrent shoulder dislocations. Journal of isakos: joint disorders \& orthopaedic sports medicine. 2018;3(4):242-8.

3. Yang JS, Mazzocca AD, Cote MP, Edgar CM, Arciero RA. Recurrent anterior shoulder instability with combined bone loss: treatment and results with the modified latarjet procedure. Am j sports med 2016;44(4):922-32. Doi: 10.1177/0363546515623929. Epub 2016 feb 1. Pmid: 26831633.

4. Garcia JC JR, Do Amaral FM, Belchior RJ, De Carvalho LQ, Markarian GG. montero efs. Comparative systematic review of fixation methods of the coracoid and conjoined tendon in the anterior glenoid to treat anterior shoulder instability. Orthop $\mathrm{j}$ sports med. 2019;7(1):2325967118820539. Doi: 10.1177/2325967118820539. Pmid: 30719477; pmcid: pmc6348521.

5. Young Aa, Maia R, Berhouet J, Walch G. Open latarjet procedure for management of bone loss in anterior instability of the glenohumeral joint. Journal of shoulder and elbow surgery 2011;20(2):s61-9

6. Mizuno N, Denard PJ, Raiss P, Melis B, Walch G. Longterm results of the latarjet procedure for anterior instability of the shoulder. Journal of shoulder and elbow surgery. 2014;23(11):1691-9.

7. Gupta A, Delaney R, Petkin K, Lafosse 1. Complications of the latarjet procedure. Current reviews in musculoskeletal medicine 2015;8(1):59-66.

8. Edwards TB, Walch G. The latarjet procedure for recurrent anterior shoulder instability: rationale and technique. Operative techniques in sports medicine 2002;10(1):25-32.

9. Goutallier D, Postel JM, Bernageau J, Lavau L, Voisin MC. Fatty muscle degeneration in cuff ruptures. Pre-and postoperative evaluation by ct scan. Clinical orthopaedics and related research 1994;1(304):78-83.

10. Samilson RL, Prieto V. Dislocation arthropathy of the shoulder. The journal of bone and joint surgery. American 1983;65(4):456-60.

11. Mchale KJ, Sanchez G, Lavery KP, Rossy WH, Sanchez A, Ferrari MB, Provencher MT. Latarjet technique for treatment of anterior shoulder instability with glenoid bone loss. Arthroscopy techniques. 2017;6(3):e791-9.

12. Gilbart MK, Gerber C. Comparison of the subjective shoulder value and the constant score. Journal of shoulder and elbow surgery 2007;16(6):717-21.

13. Constant CR, Murley AH. A clinical method of functional assessment of the shoulder. Clinical orthopaedics and related research 1987;1(214):160-4. 
14. Allain J, Goutallier D, Glorion C. Long-term results of the latarjet procedure for the treatment of anterior instability of the shoulder. Jbjs. 1998;80(6):841-52.

15. Hovelius L, Sandström B, Sundgren K, Saebö M. One hundred eighteen bristow-latarjet repairs for recurrent anterior dislocation of the shoulder prospectively followed for fifteen years: study i-clinical results. Journal of shoulder and elbow surgery 2004;13(5):509-16.

16. Coudane HW, Walch G. G. L'instabilité antérieure chronique de l'épaule chez l'adulte. Rev chir orthop. 2000;86(suppl i):91-150.

17. Schmid SL, Farshad M, Catanzaro S, Gerber C. The latarjet procedure for the treatment of recurrence of anterior instability of the shoulder after operative repair: a retrospective case series of forty-nine consecutive patients. Jbjs 2012;94(11):e75.

18. Picard F, Saragaglia D, Montbarbon E, Tourne Y, Thony F, Charbel A. Anatomo-clinical consequences of the vertical sectioning of the subscapular muscle in latarjet intervention. Revue de chirurgie orthopédique et reparatrice de l'appareil moteur 1998;84(3):217-23.

19. Maynou C, Cassagnaud $X$, Mestdagh $H$. Function of subscapularis after surgical treatment for recurrent instability of the shoulder using a bone-block procedure. The journal of bone and joint surgery. British 2005;87(8):1096-101. 